\title{
A REACTION-DIFFUSION SYSTEM ARISING IN POPULATION GENETICS
}

\author{
By \\ W. H. RUAN
}

Department of Mathematics, Computer Science and Statistics, Purdue University Calumet, Hammond, IN

Abstract. This paper is concerned with a nonweakly coupled system of parabolic equations that models the reaction-diffusion process of a population of continuously reproducing diploids with two alleles at one locus of genes. We assume that the fitnesses of genotypes are density dependent and the boundary condition is Dirichlet type with the boundary data given in the form such that the system has a unique uniform steady-state solution. The existence of nonuniform steady-state solutions is examined in relation with the stability of uniform steady-state solutions. In several cases we show that a nonuniform steady-state solution exists if the uniform one is unstable. We also investigate the stability and attractivity of the uniform steadystate solution in various cases. Some results of global convergence of solutions to a uniform steady-state solution are also given.

1. Introduction. This paper is concerned with a coupled system of reactiondiffusion equations that models a population of continuously reproducing diploids living in a bounded domain. Suppose at a locus of a certain chromosome pair there is a gene having two forms of alleles, denoted as $A$ and $a$. The population is then divided into three genotypes, $A A, A a$, and $a a$, according to the type of alleles residing at the locus. Let $\eta_{A A}, \eta_{A a}$, and $\eta_{a a}$ be the Malthusian fitness functions of the respective genotypes, let $N$ be the overall population density, and let $p$ be the frequency of the allele $A$. Then $A$ and $a$ have the allele fitness

$$
\eta_{A} \equiv p \eta_{A A}+(1-p) \eta_{A a}, \quad \eta_{a} \equiv p \eta_{A a}+(1-p) \eta_{a a},
$$

and the whole population has the mean fitness

$$
\eta \equiv p \eta_{A}+(1-p) \eta_{a}
$$

Assume that the population diffuses at the constant (unit) rate and mates at random. The reaction-diffusion process for $p$ and $N$ is governed by the following coupled system of parabolic equations:

$$
\begin{aligned}
p_{t}-\Delta p & =\left(\eta_{A}-\eta_{a}\right) p(1-p)+\frac{2}{N} \nabla N \cdot \nabla p, \quad x \in \Omega, t>0, \\
N_{t}-\Delta N & =\eta N,
\end{aligned}
$$

Received November 2, 1993.

1991 Mathematics Subject Classification. Primary 35K57, 92D10.

(C)1996 Brown University 
where $\Delta$ is the Laplacian operator and $\Omega$ is a bounded domain in $\mathbb{R}^{m}$ representing the habitat of the population. This model is suggested by Lui [7]. A special feature is that it does not assume natural selection of genes to be weak. Hence both gene evolution and population dynamics are involved in the same time frame. This consideration is based on some recent evidence in biology. Mathematically, the model is different from traditional population genetic models in that it is nonweakly coupled, that is, the equations are coupled not only through the unknown functions but also through their first-order derivatives. In general, nonweakly coupled systems are more difficult to analyze than weakly coupled ones.

The weakly coupled version of the model-dropping $(2 / N) \nabla N \cdot \nabla p$ from the first equation of (1.2) -is treated by Lui [7, 8]. Various results on the asymptotic behavior of time-dependent solutions and existence of nonuniform steady-state solutions are obtained. Some results for the nonweakly coupled systems with the homogeneous Neumann boundary condition

$$
\partial N / \partial \nu=0, \quad \partial p / \partial \nu=0 \quad \text { on } \partial \Omega
$$

are given by Lui and Selgrade [9], where the system is transformed into a weakly coupled form

$$
\begin{aligned}
u_{t}-\Delta u & =u \eta_{A}(u / N, N), \quad \text { in } \Omega \times(0, \infty), \\
N_{t}-\Delta N & =N \eta(u / N, N),
\end{aligned}
$$

by introducing the function $u=p N$, which is the density of allele $A$. It is shown that every uniform steady-state solution is an extreme point on the zero mean-fitness curve

$$
\Gamma \equiv\{(\eta, N) \in[0,1] \times(0, \infty): \eta(p, N)=0\}
$$

and vice versa. Furthermore, every local maximum point on $\Gamma$ is stable and every local minimum point on $\Gamma$ is unstable as a steady-state solution. The method in [9] is the contracting rectangles method developed in [3]. The transformation from $\{p, N\}$ to $\{u, N\}$ causes complications in implementing the method. In particular, it causes difficulty in determining the attraction regions of stable uniform steady-state solutions.

In a recent paper [13], we extended the technique of contracting rectangles to nonweakly coupled systems. The extended technique enables us to treat the model directly, without transforming it into weakly coupled form. Using this direct approach, we are able to determine the attraction regions for various uniform steady-state solutions of Problem (1.2) with the homogeneous Neumann boundary condition (1.3). In some cases, for instance in the heterozygote superior case, we even obtain global attractivity of the nontrivial steady-state solution (cf. [12]).

The purpose of this paper is to discuss Problem (1.2) with Dirichlet-type boundary condition

$$
\left.p\right|_{\partial \Omega}=p_{s},\left.\quad N\right|_{\partial \Omega}=N_{s},
$$

where $\left(p_{s}, N_{s}\right)$ is a constant solution of the equations

$$
\begin{aligned}
\left(\eta_{A}-\eta_{a}\right) p(1-p) & =0, \\
N \eta(p, N) & =0, \quad(p, N) \in[0,1] \times(0, \infty) .
\end{aligned}
$$


Hence $\left(p_{s}, N_{s}\right)$ is the only uniform steady-state solution of Problem (1.2), (1.4). Our main concern is the existence of nonuniform steady-state solutions and asymptotic behavior of time-dependent solutions. Biologically, a nonuniform steady-state solution represents a spatial heterogeneous coexistence pattern. We show that, in various cases, a nonuniform steady-state solution exists if the uniform one is unstable. We also examine the stability and attraction region of the uniform steady-state solution $\left(p_{s}, N_{s}\right)$ in all cases. Our results indicate that in contrast to the cases where the boundary condition is of homogeneous Neumann type, a local minimum point on the zero mean-fitness curve $\Gamma$ can be a stable steady-state solution. Some global convergence of solutions to the uniform steady-state solution are also given.

Throughout this paper, we assume the following condition:

The finiteness functions $\eta_{A A}, \eta_{A a}$, and $\eta_{a a}$ are continuously differentiable and monotone decreasing in $N$ with $\eta_{A A}(0), \eta_{A a}(0)$, and $\eta_{a a}(0)$ positive.

From this assumption it is easy to see that there exist positive constants $K_{A A}, K_{A a}$, and $K_{a a}$ such that

$$
\eta_{A A}\left(K_{A A}\right)=\eta_{A a}\left(K_{A a}\right)=\eta_{a a}\left(K_{a a}\right)=0 .
$$

Without loss of generality, we assume $K_{a a} \leq K_{A A}$. According to the order relations of $K_{A A}, K_{A a}$, and $K_{a a}$, the behavior of the systems are classified into three types:

Type 1. $K_{a a} \leq K_{A A}<K_{A a}$ (heterozygote superior),

Type 2. $K_{a a} \leq K_{A a} \leq K_{A A}$ (heterozygote intermediate),

Type 3. $K_{A a}<K_{a a} \leq K_{A A}$ (heterozygote inferior).

It is easy to see that $\left(0, K_{a a}\right)$ and $\left(1, K_{A A}\right)$ are solutions of $(1.5)$ for all types. Using $\left[12\right.$, Theorem 3.2] we see that there is exactly one more solution $\left(p^{*}, N^{*}\right)$ such that $0<p^{*}<1, N^{*}>0$ for systems of Types 1 and 3. Furthermore, $\left(p^{*}, N^{*}\right)$ is the only critical point on the zero mean-fitness curve $\Gamma$ : a maximum point for Type 1 and a maximum point for Type 3. Using the relation (1.1) it can be shown that $K_{A A}<N^{*}<K_{A a}$ for Type 1 and $K_{A a}<N^{*}<K_{a a}$ for Type 3. For Type 2 systems, if $K_{a a}<K_{A A}$ then $\left(0, K_{a a}\right)$ and $\left(1, K_{A A}\right)$ are the only solutions of (1.5), while if $K_{a a}=K_{A A}$ then every point $\left(p^{*}, N^{*}\right)$ with

$$
N^{*}=K_{A A}=K_{A a}=K_{a a}, \quad p^{*} \in[0,1],
$$

is a solution of (1.5).

The paper is organized as follows. In Sec. 2, we discuss the existence of nonuniform steady-state solutions and its relationship with the stability of the uniform steady-state solution $\left(p_{s}, N_{s}\right)$, where $\left(p_{s}, N_{s}\right)$ is either $\left(0, K_{a a}\right),\left(1, K_{A A}\right)$, or $\left(p^{*}, N^{*}\right)$. In Sec. 3, we determine the attraction region of the uniform steady-state solution when it is a local maximum point on $\Gamma$. In particular, we give some results on the global attractivity of $\left(p_{s}, N_{s}\right)$ for all the solutions in the set $[0,1] \times(0, \infty)$.

2. Existence of nonuniform steady-state solutions. In this section we discuss the existence of nonuniform steady-state solutions and its relationship with the stability of the uniform steady-state solution $\left(p_{s}, N_{s}\right)$, where $\left(p_{s}, N_{s}\right)$ is either $\left(0, K_{a a}\right)$, 
$\left(1, K_{A A}\right)$, or $\left(p^{*}, N^{*}\right)$. For each of these three types of $\left(p_{s}, N_{s}\right)$ we determine its stability and give a condition under which nonuniform steady-state solutions exist. The technique adopted in this section is the so-called "stability-existence" method developed in $[14,15]$, which uses the stability of certain steady-state solutions of a parabolic system in a convex invariant set to determine the existence of other steadystate solutions. Suppose $E$ is a bounded closed convex subset of the Banach space $X \equiv C(\bar{\Omega}) \oplus C(\bar{\Omega})$, and suppose that $E$ is forward invariant for the semiflow $U$ generated by the parabolic system

$$
\begin{aligned}
\partial_{t} u-L_{i} u_{i} & =f_{i}(x, \mathbf{u}, \nabla \mathbf{u}) & & \text { in } \Omega \times(0, \infty), \\
B_{i} u_{i} & =g_{i}(x) & & \text { on } \partial \Omega \times(0, \infty), \quad i=1,2, \\
u_{i}(x, 0) & =u_{i, 0}(x) & & \text { in } \Omega,
\end{aligned}
$$

where $L_{i}$ and $B_{i}$ are the uniformly elliptic operator and the boundary operator given, respectively, by

$$
L_{i}=\sum_{j, l=1}^{m} a_{j, l}^{i}(x) \frac{\partial^{2}}{\partial x_{l} \partial x_{j}}, \quad B_{i}=\alpha^{i}(x) \frac{\partial}{\partial \nu}+\beta^{i}(x),
$$

$\mathbf{u}=\left(u_{1}, u_{2}\right)$ and $f_{i}, g_{i}, a_{j, l}^{i}, \alpha^{i}, \beta^{i}$ are $C^{1}$-functions of their arguments. Let $\varphi$ be a steady-state solution of $(2.1)$ in $E$. Then for each $t>0, \varphi$ is a fixed point of the time- $t$ map $U(t): \mathbf{u}_{0} \mapsto \mathbf{u}(t)$, where $\mathbf{u}(t)$ is the solution of (2.1) with the initial data $\mathbf{u}(0)=\mathbf{u}_{0}$. The fixed point index ind $_{E}(U(t), \varphi)$ can be computed by using the theory of Amann [1] and Dancer [4]. Let $W_{\varphi}$ be the wedge defined by

$$
W_{\varphi} \equiv \operatorname{cl}\{\xi \in X ; \xi+r \varphi \in E \text { for some } r>0\}
$$

and let $S_{\varphi}$ be the largest linear subspace of $X$ contained in $W_{\varphi}$. Here "cl" denotes the topological closure of the set in $X$. It is shown in [15, Theorem 3.3] that whenever the equation

$$
L \mathbf{u}+f^{\prime}(\varphi) \mathbf{u}=0, \quad \mathbf{u} \in W_{\varphi},
$$

has no nontrivial solution, the index $\operatorname{ind}_{E}(U(t), \varphi)$ exists and takes a common integer value for all but at most a countable set of $t>0$. Here $L+f^{\prime}(\varphi)$ is the Fréchet derivative of the operator $L+f \equiv\left(L_{i}+f_{i}\right)$ at $\varphi$. Let us denote this common value by $i_{E}(\varphi)$. By using the results in [14], it can be shown that $i_{E}(\varphi)=0$ if the unstable manifold of $\varphi$ traverses $S_{\varphi}$ and $i_{E}(U, \varphi)=(-1)^{r}$ if the unstable manifold of $\varphi$ is tangent to $S_{\varphi}$ and its dimensionality in $S_{\varphi}$ is $r$. It is also shown that if $i_{E}(\varphi) \neq 1$ and if $U(t)$ is compact in $E$ for $t>0$, then Problem (2.1) admits at least one steady-state solution in $E$ different from $\varphi$. In the case in which $X$ has the direct decomposition $X=S_{\varphi} \oplus V$, for some closed linear subspace $V$, the index $i_{E}(\varphi)$ can be conveniently computed by using the following proposition.

Proposition 2.1. Suppose $U(t)$ is compact in $E$ for each $t>0$. If equation (2.2) has no nontrivial solution, then the index $i_{E}(\varphi)$ exists. Furthermore, $i_{E}(\varphi)=0$ if the operator $P\left(L+f^{\prime}(\varphi)\right)$ has an eigenvector in $P\left(W_{\varphi}\right)$ corresponding to a positive eigenvalue, and $i_{E}(U, \varphi)=(-1)^{m_{p}}$ if $P\left(L+f^{\prime}(\varphi)\right)$ has no eigenvector in $P\left(W_{\varphi}\right)$ 
corresponding to a positive eigenvalue. Here $m_{p}$ is the sum of multiplicities of positive eigenvalues of $L+f^{\prime}(\varphi)$ in $S_{\varphi}$.

A proof of this proposition is given in $[14, \S 3]$. The above results can be extended to the situation when several steady-state solutions $\left\{\varphi_{k}\right\} \in E$ are known. In this case, whenever the sum $\sum_{k} i_{E}\left(\varphi_{k}\right) \neq 1$ there exists at least one additional steady-state solution in $E$ (cf. [15, Theorem 3.5]). The compactness of $U(t)$ can be obtained if $E$ is bounded and the functions $f_{i} \equiv f_{i}(x, \mathbf{u}, \mathbf{v}), i=1,2$, satisfy the following Nagumo-type condition:

There exist positive functions $M_{1} \equiv M_{1}(r)$ and $M_{2} \equiv M_{2}(r, \rho)$ such that

$$
\begin{aligned}
& \left|f_{1}(x, \mathbf{u}, \mathbf{v})\right| \leq M_{1}\left(|\mathbf{u}|_{\mathbf{R}^{2}}\right)\left(1+\left|\mathbf{v}_{1}\right|_{\mathbf{R}^{m}}^{2}\right), \\
& \left|f_{2}(x, \mathbf{u}, \mathbf{v})\right| \leq M_{2}\left(|\mathbf{u}|_{\mathbf{R}^{2}},\left|\mathbf{v}_{1}\right|_{\mathbf{R}^{m}}\right)\left(1+\left|\mathbf{v}_{2}\right|_{\mathbf{R}^{m}}^{2}\right)
\end{aligned}
$$

for all $x \in \bar{\Omega}, \mathbf{u} \in \mathbf{R}^{2}$, and $\mathbf{v} \equiv\left(\mathbf{v}_{1}, \mathbf{v}_{2}\right) \in \mathbf{R}^{2 m}$.

In fact, it is shown in [13, Theorem 2.1] that for each $\mu>0, \delta>0$, the $C^{1+\mu}$ norm of the solution $\mathbf{u}(t)$ in $E$ is uniformly bounded. The set $E$ can often be defined by time-independent upper and lower solutions $\tilde{\mathbf{u}} \equiv\left(\tilde{u}_{1}, \tilde{u}_{2}\right), \hat{\mathbf{u}} \equiv\left(\hat{u}_{1}, \hat{u}_{2}\right)$ such that

$$
E=\left\{\left(u_{1}, u_{2}\right) \in X: \hat{u}_{1} \leq u_{1} \leq \tilde{u}_{1}, \hat{u}_{2} \leq u_{2} \leq \tilde{u}_{2}, \text { in } \Omega\right\} .
$$

Here $\hat{u}_{i}, \tilde{u}_{i}$ are coupled upper and lower solutions satisfying the relations $\hat{u}_{i}(x) \leq$ $\tilde{u}_{i}(x)$ and the differential inequalities

$$
\begin{array}{r}
-L_{i} \hat{u}_{i} \leq f_{i}\left(x, u_{1}, \ldots, \hat{u}_{i}, \ldots, u_{n}, \nabla u_{1}, \ldots, \nabla \hat{u}_{i}, \ldots, \nabla u_{n}\right), \\
\text { in } \Omega
\end{array}
$$

where $u_{i}, i=1,2$, are arbitrary functions satisfying the relation

$$
\hat{u}_{i}(x) \leq u_{i}(x) \leq \tilde{u}_{i}(x), \quad x \in \Omega, i=1,2 .
$$

It is well known that the set $E$ defined in this way is a bounded closed convex invariant set for Problem (2.1).

For our population genetic model (1.2), (1.4), the above Nagumo condition is obviously satisfied. In this case, a pair of time-independent functions $(\tilde{p}, \widetilde{N}),(\hat{p}, \widehat{N})$ are coupled upper and lower solutions if they satisfy the inequalities $\hat{p} \leq \tilde{p}, \hat{N} \leq \widetilde{N}$ in $\Omega$ and

$$
\begin{aligned}
-\Delta \tilde{p} & \geq\left(\eta_{A}-\eta_{a}\right)(\tilde{p}, N) \tilde{p}(1-\tilde{p})+\frac{2}{N} \nabla N \cdot \nabla \tilde{p}, \\
-\Delta \hat{p} & \leq\left(\eta_{A}-\eta_{a}\right)(\hat{p}, N) \hat{p}(1-\hat{p})+\frac{2}{N} \nabla N \cdot \nabla \hat{p}, \quad \text { in } \Omega, \\
-\Delta \tilde{N} & \geq \tilde{N} \eta(p, \tilde{N}), \\
-\Delta \widehat{N} & \leq \widehat{N} \eta(p, \widehat{N}), \\
\left.\hat{p}\right|_{\partial \Omega} & \leq p_{s} \leq\left.\tilde{p}\right|_{\partial \Omega},\left.\quad \hat{N}\right|_{\partial \Omega} \leq N_{s} \leq\left.\tilde{N}\right|_{\partial \Omega},
\end{aligned}
$$


where $N$ and $p$ are arbitrary $C^{1}$-functions satisfying

$$
\widehat{N} \leq N \leq \tilde{N}, \quad \hat{p} \leq p \leq \tilde{p} \quad \text { in } \Omega .
$$

If $\hat{p}, \tilde{p}, \widehat{N}, \tilde{N}$ are constants, then $(2.3)$ is reduced to

$$
\begin{aligned}
& \left(\eta_{A}-\eta_{a}\right)(\tilde{p}, N) \tilde{p}(1-\tilde{p}) \leq 0, \\
& \left(\eta_{A}-\eta_{a}\right)(\hat{p}, N) \hat{p}(1-\hat{p}) \geq 0, \\
& \tilde{N} \eta(p, \tilde{N}) \leq 0, \quad \text { for }(p, N) \in[\hat{p}, \tilde{p}] \times(\widehat{N}, \tilde{N}) . \\
& \hat{p} \leq p_{s} \leq \tilde{p}, \quad \widehat{N} \leq N_{s} \leq \tilde{N},
\end{aligned}
$$

We call such a rectangle $\Sigma=[\hat{p}, \tilde{p}] \times[\widehat{N}, \tilde{N}]$ an invariant rectangle. To obtain the existence of nonuniform steady-state solutions, we first construct an invariant rectangle that contains the uniform steady-state solution $\varphi \equiv\left(p_{s}, N_{s}\right)$ and then analyze the eigenvalues and eigenvectors of the linearized operator $L+f^{\prime}(\varphi)$ in relation with $W_{\varphi}$ and $S_{\varphi}$ by using Proposition 2.1. In constructing invariant rectangles, we need certain properties of the functions $\eta_{A}, \eta_{a}$, and $\eta$. First, by using assumption (A) we see that $\eta_{A A}, \eta_{A a}$, and $\eta_{a a}$ are decreasing in $N$. Hence $\eta_{A}, \eta_{a}$, and $\eta$ are also decreasing in $N$. This implies that there exist continuous functions $\phi_{A}, \phi_{a}$, and $\phi$ defined on $p \in[0,1]$ such that

$$
\eta_{A}\left(p, \phi_{A}(p)\right)=0, \quad \eta_{a}\left(p, \phi_{a}(p)\right)=0, \quad \eta(p, \phi(p))=0
$$

and

$$
\phi(0)=\phi_{a}(0)=K_{a a}, \quad \phi(1)=\phi_{A}(1)=K_{A A}, \quad \phi_{A}(0)=\phi_{a}(1)=K_{A a} .
$$

Furthermore, if $N<\phi(p)$ then $\eta(p, N)>0$, and if $N>\phi(p)$ then $\eta(p, N)<0$. The same is true for $\phi_{A}$ and $\phi_{a}$. The monotonicity of these functions given in the following lemma plays an important role in the discussion below.

Lemma 2.1. Let (A) hold. Then

(i) for type 1 systems, $\phi_{A}$ is monotone decreasing, $\phi_{a}$ is monotone increasing, and $\phi$ has a unique maximum point $\left(p^{*}, N^{*}\right)$ in $(0,1) \times(0, \infty)$,

(ii) for type 2 systems, $\phi_{A}, \phi_{a}$, and $\phi$ are monotone increasing unless the respective relation of $K_{A a}=K_{A A}, K_{a a}=K_{A a}$, and $K_{a a}=K_{A a}=K_{A A}$ holds; if a relation does hold, the corresponding function is constant,

(iii) for type 3 systems, $\phi_{A}$ is monotone increasing, $\phi_{a}$ is monotone decreasing, and $\phi$ has a unique minimum point $\left(p^{*}, N^{*}\right)$ in $(0,1) \times(0, \infty)$.

A proof of the lemma can be found in [12] under the condition that $\eta_{A A}^{\prime}, \eta_{A a}^{\prime}, \eta_{a a}^{\prime}$ are strictly negative. With some minor modification in the proof, this condition can be replaced by the strict monotone decreasing property of $\eta_{A A}, \eta_{A a}$, and $\eta_{a a}$.

Since $\left(p_{s}, N_{s}\right)$ is a constant solution, it is easy to see that the linearized operator $A \equiv L+f^{\prime}(\varphi)$ at $\varphi=\left(p_{s}, N_{s}\right)$ takes the form

$$
A(\xi, \zeta) \equiv\left(\Delta \xi+a_{11} \xi+a_{12} \zeta, \Delta \zeta+a_{21} \xi+a_{22} \zeta\right)
$$


where

$$
\begin{aligned}
& a_{11}=p_{s}\left(1-p_{s}\right)\left(\eta_{A A}-2 \eta_{A a}+\eta_{a a}\right)\left(N_{s}\right)+\left(1-2 p_{s}\right)\left(\eta_{A}-\eta_{a}\right)\left(p_{s}, N_{s}\right), \\
& a_{12}=p_{s}\left(1-p_{s}\right) \partial_{N}\left(\eta_{A}-\eta_{a}\right)\left(p_{s}, N_{s}\right), \\
& a_{21}=N_{s}\left(\eta_{A}-\eta_{a}\right)\left(p_{s}, N_{s}\right), \\
& a_{22}=\eta\left(p_{s}, N_{s}\right)+N_{s} \partial_{N} \eta\left(p_{s}, N_{s}\right) .
\end{aligned}
$$

Hence an eigenvector $(\xi, \zeta)$ and its corresponding eigenvalue $\lambda$ satisfy the elliptic problem

$$
\begin{gathered}
\Delta \xi+a_{11} \xi+a_{12} \zeta=\lambda \xi, \quad \text { in } \Omega, \\
\Delta \zeta+a_{21} \xi+a_{22} \zeta=\lambda \zeta, \\
\left.\xi\right|_{\partial \Omega}=0,\left.\quad \zeta\right|_{\partial \Omega}=0 .
\end{gathered}
$$

By the theory of linearization, the steady-state solution $\varphi=\left(p_{s}, N_{s}\right)$ is unstable if the above eigenvalue problem has an eigenvalue with positive real part. Let $\mu_{1}, \mu_{2}$ be the eigenvalues of the matrix $\left(a_{i j}\right)$ with $\operatorname{Re} \mu_{1} \geq \operatorname{Re} \mu_{2}$, and let $\lambda_{0}$ be the first eigenvalue of $-\Delta$ in $\Omega$ with the homogeneous Dirichlet boundary condition. Then $\lambda_{0}>0$ and $\mu_{1}-\lambda_{0}$ is the eigenvalue of (2.5) that has the maximum real part. Hence $\left(p_{s}, N_{s}\right)$ is unstable if $\operatorname{Re} \mu_{1}>\lambda_{0}$ and is asymptotically stable if $\operatorname{Re} \mu_{1}<\lambda_{0}$.

We now consider the existence of a nonuniform steady-state solution for each case. First consider the case where $\left(p_{s}, N_{s}\right)=\left(0, K_{a a}\right)$. We show that whenever $\left(p_{s}, N_{s}\right)$ is unstable then there exists at least one nonuniform steady-state solution. First, by computation, $a_{i j}$ can be found as

$$
\begin{aligned}
& a_{11}=\left(\eta_{A}-\eta_{a}\right)\left(0, K_{a a}\right)=\eta_{A a}\left(K_{a a}\right), \quad a_{12}=0, \\
& a_{21}=K_{a a} \eta_{A a}\left(K_{a a}\right), \quad a_{22}=K_{a a} \eta_{a a}^{\prime}\left(K_{a a}\right) .
\end{aligned}
$$

Hence $\eta_{A a}\left(K_{a a}\right)$ and $K_{a a} \eta_{a a}^{\prime}\left(K_{a a}\right)$ are the two eigenvalues of the matrix $\left(a_{i j}\right)$. Since by assumption $(\mathrm{A}), \eta_{a a}^{\prime}\left(K_{a a}\right) \leq 0$, it follows that $\left(p_{s}, N_{s}\right)$ is unstable if and only if

$$
\eta_{A a}\left(K_{a a}\right)>\lambda_{0} \text {. }
$$

The following theorem shows that a nonuniform steady-state solution exists if the above condition holds.

Theorem 2.1. Let (A) and (2.7) hold. Suppose $\left(p_{s}, N_{s}\right)=\left(0, K_{a a}\right)$. Then Problem (1.2), (1.4) has a nonconstant steady-state solution in the set $\Sigma=[0,1] \times\left[K_{a a}, K\right]$, where $K=N^{*}$ for type 1 systems, and $K=K_{A A}$ for type 2 systems.

Proof. Since (2.7) implies $\eta_{A a}\left(K_{a a}\right)>0$, it follows from condition (A) that $K_{a a}<$ $K_{A a}$. Hence the system is of either type 1 or type 2. From Lemma 2.1 we see that $K_{a a}$ and $K$ are the minimum and maximum values of $N$ on the zero meanfitness curve $\Gamma$ respectively. In view of assumption (A) and the definition of $\eta$, $\eta(p, N)<0$ if $(p, N)$ is above $\Gamma$ and $\eta(p, N)>0$ if $(p, N)$ is below $\Gamma$. Hence $\widehat{N}=K_{a a}$ and $\tilde{N}=K$ satisfy the related inequalities in (2.4). Since $\hat{p}=0, \tilde{p}=1$ obviously satisfy the corresponding inequalities in $(2.4), \Sigma$ is an invariant rectangle and $\varphi=\left(0, K_{a a}\right) \in \Sigma$. By computation

$$
W_{\varphi}=\{(\xi, \zeta) \in X: \xi \geq 0, \zeta \geq 0\}, \quad S_{\varphi}=\{0\} .
$$


The second relation implies that the operator $P$ is the identity operator in $X$. Hence by Proposition $2.1, i_{\Sigma}(\varphi)$ exists and equals 0 if $(2.5)$ has no nontrivial solution in $W_{\varphi}$ for $\lambda=0$, and has nontrivial solutions for some $\lambda>0$. Since by (2.6) and (2.7), $a_{11}>\lambda_{0}, a_{12}=0$, it follows from the Krein-Rutman Theorem that Problem (2.5) has a nontrivial solution in $W_{\varphi}$ if and only if $\lambda=a_{11}-\lambda_{0}>0$. Therefore by Proposition 2.1 and [15, Theorem 3.5], Problem (1.2), (1.4) has at least one steady-state solution in $\Sigma$ different from the uniform solution $\left(p_{s}, N_{s}\right)$. The proof is complete. Q.E.D.

We next consider the case where $\left(p_{s}, N_{s}\right)=\left(1, K_{A A}\right)$. Similar to the previous case, we show that Problem (1.2), (1.4) has at least one nonuniform steady-state solution if the uniform steady-state solution is unstable. By computation

$$
\begin{aligned}
& a_{11}=-\left(\eta_{A}-\eta_{a}\right)\left(1, K_{A A}\right)=\eta_{A a}\left(K_{A A}\right), \quad a_{12}=0, \\
& a_{21}=-K_{A A} \eta_{A a}\left(K_{A A}\right), \quad a_{22}=K_{A A} \eta_{A A}^{\prime}\left(K_{A A}\right) .
\end{aligned}
$$

Since $\eta_{A A}^{\prime} \leq 0$, it follows that $\left(p_{s}, N_{s}\right)$ is unstable whenever

$$
\eta_{A a}\left(K_{A A}\right)>\lambda_{0} \text {. }
$$

In this case we have

Theorem 2.2. Suppose (A) holds and suppose $\left(p_{s}, N_{s}\right)=\left(1, K_{A A}\right)$. If $(2.9)$ holds then Problem (1.2), (1.4) has a nonuniform steady-state solution in the rectangle $\Sigma=[0,1] \times\left[K_{a a}, N^{*}\right]$.

Proof. Since $\eta_{A a}\left(K_{A A}\right)>\lambda_{0}>0$, it follows from assumption (A) that $K_{A a}>K_{A A}$. This only occurs in type 1 systems. In view of Lemma $2.1,\left(p^{*}, N^{*}\right)$ is the unique maximum point on the zero mean-fitness curve $\Gamma$. Thus $N^{*}$ and $K_{a a}$ are the maximum and minimum values of $N$ on $\Gamma$ respectively. Hence, as in the proof of Theorem 2.1, $\Sigma$ is invariant. It is easy to see that

$$
W_{\varphi}=\{(\xi, \zeta) \in X: \xi \leq 0\}, \quad S_{\varphi}=\{(0, \zeta): \zeta \in C(\bar{\Omega})\} .
$$

This implies that the operator $P$ satisfies

$$
P(\xi, \zeta)=(\xi, 0) \text { for }(\xi, \zeta) \in X .
$$

We use Proposition 2.1 to show that $i_{\Sigma}(\varphi)$ exists and equals 0 . For this purpose, we verify that (1) Problem (2.5) has no nontrivial solution in $W_{\varphi}$ for $\lambda=0$, and (2) $P A$ has an eigenvector in $P\left(W_{\varphi}\right)=\{(\xi, 0): \xi \leq 0\}$ for some $\lambda>0$. In view of (2.8) and (2.9), $a_{11}>\lambda_{0}$ and $a_{12}=0$. Hence by the Krein-Rutman Theorem, Problem (2.5) has no solution with nonpositive nontrivial $\xi$ component if $\lambda=0$. Thus any solution of $\left(2.5\right.$; with $\lambda=0$ in $W_{\varphi}$ has the form $(0, \zeta)$. However, by assumption (A), $a_{22} \equiv K_{A A} \eta_{A A}^{\prime}\left(K_{A A}\right)<0$. It follows that whenever $\xi$ is zero, $\zeta$ is also zero. This implies that Problem (2.5) has no nontrivial solution in $W_{\varphi}$ if $\lambda=0$. Consider the eigenvalue problem of the operator $P A$ in $P\left(W_{\varphi}\right)$. It takes the form

$$
\Delta \xi+a_{11} \xi=\lambda \xi, \quad \xi \leq 0 \quad \text { in } \Omega .
$$

Since $a_{11}>\lambda_{0}$, the problem has negative solutions for $\lambda=a_{11}-\lambda_{0}$. Therefore, by Proposition 2.1 and [15, Theorem 3.5], Problem (1.2), (1.4) has at least one steadystate solution in $\Sigma$. The proof is complete. Q.E.D. 
REMARK. The same method can be used with different invariant rectangles. For example, if $\Sigma=[\underline{p}, 1] \times\left[K_{A A}, N^{*}\right]$, then it can be shown that $\Sigma$ is also an invariant rectangle. Here $\underline{p}$ is a point such that $0 \leq \underline{p}<p^{*}$ and $\eta\left(\underline{p}, K_{A A}\right)=0$. The existence of such $\underline{p}$ follows from the intermediate value theorem and the relations $\phi(0)=K_{a a} \leq K_{A A}, \phi\left(p^{*}\right)=N^{*}>K_{A A}$. In this case

$$
W_{\varphi}=\{(\xi, \zeta) \in X: \xi \leq 0, \zeta \geq 0\}, \quad S_{\varphi}=\{0\} .
$$

Using a similar argument as in the proof of Theorem 2.1, we can show that there is a nonuniform steady-state solution in $\Sigma$.

We consider the case $\left(p_{s}, N_{s}\right)=\left(p^{*}, N^{*}\right)$, where $\left(p^{*}, N^{*}\right)$ is a solution of (1.5). The constants $a_{i j}$ are

$$
\begin{aligned}
& a_{11}=p^{*}\left(1-p^{*}\right)\left(\eta_{A A}-2 \eta_{A a}+\eta_{a a}\right)\left(N^{*}\right), \\
& a_{12}=p^{*}\left(1-p^{*}\right) \partial_{N}\left(\eta_{A}-\eta_{a}\right)\left(p^{*}, N^{*}\right), \\
& a_{21}=0, \quad a_{22}=N^{*} \partial_{N} \eta\left(p^{*}, N^{*}\right) .
\end{aligned}
$$

Since by assumption (A), $a_{22} \leq 0$, it follows that $\left(p_{s}, N_{s}\right)$ is unstable if and only if $a_{11}>\lambda_{0}$. A simplified form of $a_{11}$ is obtained by using (1.5) and the relations $p^{*} \in(0,1), N^{*}>0$, which imply

$$
\eta\left(p^{*}, N^{*}\right)=\eta_{A}\left(p^{*}, N^{*}\right)=\eta_{a}\left(p^{*}, N^{*}\right)=0 .
$$

Hence from (1.1)

$$
\begin{aligned}
0 & =\left(1-p^{*}\right) \eta_{A}+p^{*} \eta_{a} \\
& =\left(1-p^{*}\right) p^{*} \eta_{A A}+p^{*}\left(1-p^{*}\right) \eta_{a a}+\left(1-2 p^{*}+2\left(p^{*}\right)^{2}\right) \eta_{A a} .
\end{aligned}
$$

It follows that

$$
a_{11} \equiv\left(1-p^{*}\right) p^{*}\left(\eta_{A A}-2 \eta_{A a}+\eta_{a a}\right)\left(N^{*}\right)=-\eta_{A a}\left(N^{*}\right) .
$$

Thus a necessary and sufficient condition for $\left(p_{s}, N_{s}\right)$ to be unstable is

$$
-\eta_{A a}\left(N^{*}\right)>\lambda_{0} \text {. }
$$

It is not clear whether the above condition implies the existence of nonuniform steady-state solutions. There are various partial results. For example, by using the stability-existence method with the invariant rectangle $\Sigma=[0,1] \times\left[N^{*}, K_{A A}\right]$, it can be shown that at least one nonuniform steady-state solution exists if the eigenvalue problem

$$
\Delta \xi-\eta_{A a}\left(N^{*}\right) \xi=\lambda \xi \quad \text { in } \Omega,\left.\quad \xi\right|_{\partial \Omega}=0
$$

has positive eigenvalues whose sum of multiplicities is odd. The details can be found in [14]. Another result is given in the next theorem.

Theorem 2.3. Let (A) and (2.13) hold. Suppose $\left(p_{s}, N_{s}\right)=\left(p^{*}, N^{*}\right)$. If

$$
\eta_{A}\left(p^{*}, N\right) \neq \eta_{a}\left(p^{*}, N\right) \text { for } N^{*}<N \leq K_{A A}
$$

then Problem (1.2), (1.4) has at least one nonuniform steady-state solution. 
Proof. We prove the theorem in the case where $\eta_{A}\left(p^{*}, N\right)>\eta_{a}\left(p^{*}, N\right)$ for $N \in$ $\left(N^{*}, K_{A A}\right]$. The other case can be treated analogously. Let $\Sigma=\left[p^{*}, 1\right] \times\left[N^{*}, K_{A A}\right]$. It is easy to verify that the constants $\hat{p}=p^{*}, \tilde{p}=1, \widehat{N}=N^{*}, \widetilde{N}=K_{A A}$ satisfy (2.4). Hence $\Sigma$ is an invariant rectangle. Furthermore, by direct computation, we see that

$$
W_{\varphi}=\{(\xi, \zeta) \in X: \xi \geq 0, \zeta \geq 0\}, \quad S_{\varphi}=\{0\} .
$$

Hence, as in the proof of Theorem 2.1 , the operator $P$ is the identity operator in $X$. We show that the operator $A$ has an eigenvector in $W_{\varphi}$ if and only if $\lambda=a_{11}-\lambda_{0}$. Once this is shown, Proposition 2.1 will imply that $i_{\Sigma}(\varphi)=0$, and the existence result follows from [15, Theorem 3.5]. Since by (2.10) and assumption (A), $a_{21}=0$ and $a_{22} \leq 0$, it follows from the Krein-Rutman Theorem that the second equation in (2.5) has only the zero solution for any nonnegative $\lambda$. Thus (2.5) reduces to the scalar elliptic problem

$$
\Delta \xi+a_{11} \xi=\lambda \xi \quad \text { in } \Omega,\left.\quad \xi\right|_{\partial \Omega}=0 .
$$

Using the relation $a_{11}>\lambda_{0}$, it can be seen that the above problem has no nontrivial nonnegative solution if $\lambda=0$, but has a positive solution if $\lambda=a_{11}-\lambda_{0}$. In view of Proposition 2.1 and [15, Theorem 3.5], Problem (1.2), (1.4) has at least one steady-state solution in $\Sigma$. The proof is complete. Q.E.D.

The above analysis also gives the stability of the uniform steady-state solution $\left(p_{s}, N_{s}\right)$ for each type of system. For type 1 systems, if $\left(p_{s}, N_{s}\right)=\left(p^{*}, N^{*}\right)$, then it is always asymptotically stable since $\eta_{A a}\left(N^{*}\right)>0$ by the relation $N^{*}<K_{A a}$, whereas if $\left(p_{s}, N_{s}\right)$ is either $\left(0, K_{a a}\right)$ or $\left(1, K_{A A}\right)$, then it is asymptotically stable if and only if the reversed relation of (2.7) or (2.9) holds respectively. For type 2 systems, if $\left(p_{s}, N_{s}\right)=\left(1, K_{A A}\right)$ then it is always asymptotically stable, since $\eta_{A a}\left(K_{A A}\right) \leq 0$. But if $\left(p_{s}, N_{s}\right)=\left(0, K_{a a}\right)$, then it is asymptotically stable if and only if the reversed relation of (2.9) holds, which occurs only if $K_{a a}<K_{A A}$. In the case $K_{a a}=K_{A A}$, there follows

$$
\eta_{a a}\left(N^{*}\right)=\eta_{A a}\left(N^{*}\right)=\eta_{A A}\left(N^{*}\right)=0<\lambda_{0} .
$$

Hence none of the conditions (2.7), (2.9), (2.13) holds and $\left(p_{s}, N_{s}\right)$ is always asymptotically stable. For type 3 systems, if $\left(p_{s}, N_{s}\right)$ is either $\left(0, K_{a a}\right)$ or $\left(1, K_{A A}\right)$, then it is asymptotically stable because in this case

$$
\eta_{A a}\left(K_{a a}\right) \leq 0, \quad \eta_{A a}\left(K_{A A}\right) \leq 0 .
$$

Finally, if $\left(p_{s}, N_{s}\right)=\left(p^{*}, N^{*}\right)$, then it is asymptotically stable if and only if the reversed relation of (2.13) holds. These results show that the stability of the uniform steady-state solution $\left(p_{s}, N_{s}\right)$ of Problem (1.2) with the Dirichlet boundary condition (1.4) is different from the stability of the same steady-state solution when the boundary condition is homogeneous Neumann type. For the homogeneous Neumann boundary condition the steady-state solution is always unstable if it is a local minimum point on $\Gamma$ (cf. [12]), but for the Dirichlet boundary condition (1.4) it can be stable. 
3. Convergence to uniform steady-state solutions. In this section we consider the asymptotic behavior of solutions of Problem (1.2), (1.4); in particular, we discuss the convergence of solutions to the uniform steady-state solution $\left(p_{s}, N_{s}\right)$ when it is a local maximum point on $\Gamma$. Throughout this section we assume that $(p, N)$ is a solution of Problem (1.2), (1.4) with the initial data $\left(p_{0}, N_{0}\right) \in[0,1] \times(0, \infty)$. Our main tool is the extended method of contracting rectangles. More precisely, we use the notion of minimum invariant rectangles defined as follows. In an invariant rectangle $\Sigma=[\hat{p}, \tilde{p}] \times[\widehat{N}, \tilde{N}], \hat{p}$ is called nonstrict if either $\hat{p}=p_{s}$ or

$$
\left(\eta_{A}-\eta_{a}\right)(\hat{p}, N) \hat{p}(1-\hat{p})=0 \text { for some } N \in[\widehat{N}, \tilde{N}] .
$$

Similarly, $\widehat{N}$ is called nonstrict if either $\widehat{N}=N_{s}$ or

$$
\widehat{N} \eta(p, \widehat{N})=0 \text { for some } p \in[\hat{p}, \tilde{p}] .
$$

The nonstrictness of $\tilde{p}$ and $\tilde{N}$ is defined analogously. The invariant rectangle $\Sigma$ is called minimum if each of $\hat{p}, \tilde{p}, \widehat{N}, \widetilde{N}$ is nonstrict. The next theorem asserts that every trajectory $(p(\cdot, t), N(\cdot, t))$ starting in an invariant rectangle has its $\omega$-limit set lie in a minimum invariant rectangle.

TheOREM 3.1. Let $\Sigma^{0}=\left[\hat{p}^{0}, \tilde{p}^{0}\right] \times\left[\widehat{N}^{0}, \widetilde{N}^{0}\right]$ be an invariant rectangle for problem (1.2), (1.4). Then there exists a minimum invariant rectangle

$$
\Sigma^{\prime} \equiv\left[\hat{p}^{\prime}, \tilde{p}^{\prime}\right] \times\left[\widehat{N}^{\prime}, \tilde{N}^{\prime}\right] \subset \Sigma^{0}
$$

such that every solution $(p(\cdot, t), N(\cdot, t))$ of $(1.2),(1.4)$ with $(p(\cdot, 0), N(\cdot, 0)) \in$ $\Sigma^{0}$ has its $\omega$-limit set contained in $\Sigma^{\prime}$.

Proof. The proof is similar to the proof of Theorem 2.1 of [12]. We use Theorem 3.1 of [13], which states the following conclusion. Suppose $\Sigma(\lambda)=[\hat{p}(\lambda), \tilde{p}(\lambda)] \times$ $[\widehat{N}(\lambda), \widetilde{N}(\lambda)], \lambda \in[0,1)$, is a family of invariant rectangles that satisfies the following condition $(\mathrm{B})$ :

The functions $\lambda \rightarrow(\hat{p}(\lambda), \widehat{N}(\lambda))$ and $\lambda \mapsto(\tilde{p}(\lambda), \tilde{N}(\lambda))$ are differentiable for $\lambda \in[0,1)$; the former is monotone nondecreasing and the latter is monotone nonincreasing in $\lambda$. If any one of the functions $\hat{p}(\lambda), \tilde{p}(\lambda), \widehat{N}(\lambda), \tilde{N}(\lambda)$ is nonstrict with respect to $\Sigma(\lambda)$ for some $\lambda \in[0,1)$, then this function is independent of $\lambda \in[0,1)$.

Under this condition, every solution $(p(\cdot, t), N(\cdot, t))$ of $(1.2),(1.4)$ whose initial data satisfies $(p(\cdot, 0), N(\cdot, 0)) \in \Sigma(0)$ enters into each $\Sigma(\lambda)$ in finite time. Namely, for each $\lambda \in[0,1)$ there exists a positive constant $T_{\lambda}$ such that

$$
(p(\cdot, t), N(\cdot, t)) \in \Sigma(\lambda) \text { for all } t \geq T_{\lambda} .
$$

We first define a set of invariant rectangles $\mathscr{S}$ by

$$
\mathscr{S}=\left\{[\hat{p}, \tilde{p}] \times[\widehat{N}, \tilde{N}] \subseteq \Sigma^{0}: \hat{p}, \tilde{p}, \widehat{N}, \tilde{N} \text { satisfy }(2.4)\right\}
$$


Define an order relation " $\prec$ " in $\mathscr{S}$ as follows:

$$
\Sigma^{1} \prec \Sigma^{2} \text { if } \Sigma^{1} \subseteq \Sigma^{2} \text { and for each initial function }\left(p_{0}, N_{0}\right) \text { satisfying }
$$

$$
\left(p_{0}(x), N_{0}(x)\right) \in \Sigma^{2} \quad \text { for } x \in \bar{\Omega}
$$

the $\omega$-limit set $\omega\left(p_{0}, N_{0}\right)$ of the solution $(p(\cdot, t), N(\cdot, t))$ lies in $\Sigma^{1}$.

It is easy to see that this relation defines a partial ordering in $\mathscr{S}$. The conclusion of the theorem will follow if we show that there exists a minimum invariant rectangle $\Sigma^{\prime} \in \mathscr{S}$ such that $\Sigma^{\prime} \prec \Sigma^{0}$. Recall that a chain $\left\{\Sigma^{\lambda} ; \lambda \in I\right\}$ of $\mathscr{S}$ is a subset of $\mathscr{S}$ with an index set $I$ such that for any pair $\lambda, \mu \in I$, either $\Sigma^{\lambda} \prec \Sigma^{\mu}$ or $\Sigma^{\mu} \prec \Sigma^{\lambda}$ holds. We first show that every chain $\mathscr{C}=\left\{\Sigma^{\lambda} ; \lambda \in I\right\}$ of $\mathscr{S}$ has a lower bound $\Sigma^{*}$ in $\mathscr{S}$ (i.e., $\Sigma^{*} \prec \Sigma^{\lambda}$ for all $\Sigma^{\lambda} \in \mathscr{C}$ ). Let $\Sigma^{\lambda}$ be written as

$$
\Sigma^{\lambda}=\left[\hat{p}^{\lambda}, \tilde{p}^{\lambda}\right] \times\left[\widehat{N}^{\lambda}, \tilde{N}^{\lambda}\right], \quad \lambda \in I,
$$

and let $\Sigma^{*}=\left[\hat{p}^{*}, \tilde{p}^{*}\right] \times\left[\widehat{N}^{*}, \widetilde{N}^{*}\right]$ be the rectangle defined by

$$
\hat{p}^{*}=\sup \left\{\hat{p}^{\lambda}\right\}, \quad \tilde{p}^{*}=\inf \left\{\tilde{p}^{\lambda}\right\}, \quad \widehat{N}^{*}=\sup \left\{\widehat{N}^{\lambda}\right\}, \quad \tilde{N}^{*}=\inf \left\{\tilde{N}^{\lambda}\right\}, \quad \lambda \in I .
$$

We show that $\Sigma^{*}$ is a lower bound for chain $\mathscr{C}$. It is clear that $\Sigma^{*} \subseteq \Sigma^{\lambda}$ for all $\Sigma^{\lambda} \in \mathscr{C}$. Since for each $\lambda \in I, \hat{p}^{\lambda}, \tilde{p}^{\lambda}, \widehat{N}^{\lambda}, \tilde{N}^{\lambda}$ satisfy (2.4), it follows from the continuity of $\eta_{A}$ and $\eta_{a}$ that $\hat{p}^{*}, \tilde{p}^{*}, \widehat{N}^{*}, \widetilde{N}^{*}$ also satisfy (2.4). Hence $\Sigma^{*} \in$ $\mathscr{S}$. It remains to show that for each $\Sigma^{\lambda} \in \mathscr{C}$ and each initial function $\left(p_{0}, N_{0}\right)$ satisfying $\left(p_{0}, N_{0}\right) \in \Sigma^{\lambda}$, the $\omega$-lilmit set $\omega\left(p_{0}, N_{0}\right)$ lies in $\Sigma^{*}$. Recall that if the orbit $\{(p(\cdot, t), N(\cdot, t)): t \geq \delta\}$ is compact for some $\delta>0$, then $\omega\left(p_{0}, N_{0}\right)$ is a compact, connected set that has the property that for any $\varepsilon>0$ there exists $T_{\varepsilon}>0$ such that the distance from $\left(p\left(\cdot, T_{\varepsilon}\right), N\left(\cdot, T_{\varepsilon}\right)\right)$ to $\omega\left(p_{0}, N_{0}\right)$ is less than $\varepsilon$ in the topology of $X \equiv C(\bar{\Omega}) \oplus C(\bar{\Omega})$. Furthermore the $\omega$-limit set is invariant in the sense that if $\left(p_{1}, N_{1}\right) \in \omega\left(p_{0}, N_{0}\right)$ and $\left(p^{\prime}, N^{\prime}\right)$ is the solution of (1.2), (1.4) with $\left(p^{\prime}(\cdot, 0), N^{\prime}(\cdot, 0)\right)=\left(p_{1}, N_{1}\right)$, then $\left(p^{\prime}(\cdot, t), N^{\prime}(\cdot, t)\right) \in \omega\left(p_{0}, N_{0}\right)$ for all $t \in \mathbb{R}$ (cf. [17]). In view of Lemma 3.1 and the imbedding relationship (2.2) and (2.3) of [13], the orbit $\{(p(\cdot, t), N(\cdot, t)): t \geq 1\}$ is bounded in $C^{1+\delta}(\bar{\Omega}) \oplus C^{1+\delta}(\bar{\Omega})$ for some $\delta>0$. Hence, every orbit in $\Sigma^{0}$ is compact in $X$, and $\omega\left(p_{0}, N_{0}\right)$ exists and has the above properties. We now show that

$$
\omega\left(p_{0}, N_{0}\right) \subseteq \Sigma^{*} .
$$

Suppose by contradiction that (3.1) is not true. Then for some $\Sigma^{\mu} \in \mathscr{C}$ and some initial function $\left(p_{0}, N_{0}\right) \in \Sigma^{\mu}$ there exist $\left(p^{\prime}, N^{\prime}\right) \in \omega\left(p_{0}, N_{0}\right)$ and $x_{0} \in \bar{\Omega}$ such that $\left(p^{\prime}\left(x_{0}\right), N^{\prime}\left(x_{0}\right)\right) \notin \Sigma^{*}$. Without loss of generality, we may assume that $p^{\prime}\left(x_{0}\right)<\hat{p}^{*}$. Hence, there is $\lambda \in I$ such that $p^{\prime}\left(x_{0}\right)<\hat{p}^{\lambda}$. Since $\left(p^{\prime}(x), N^{\prime}(x)\right) \in \Sigma^{\mu}$ by the invariance of $\Sigma^{\mu}$, it follows that $\hat{p}^{\mu} \leq p^{\prime}\left(x_{0}\right)<\hat{p}^{\lambda}$. Therefore, by using the fact that $\Sigma^{\mu}$ and $\Sigma^{\lambda}$ are comparable, we have $\Sigma^{\lambda} \subseteq \Sigma^{\mu}$ and consequently $\Sigma^{\lambda} \prec \Sigma^{\mu}$. But this leads to $\left(p^{\prime}(x), N^{\prime}(x)\right) \in \Sigma^{\lambda}$ for all $x \in \bar{\Omega}$, contradicting the relation $p^{\prime}\left(x_{0}\right)<\hat{p}^{\lambda}$. This proves the relation (3.1), which implies that $\Sigma^{*}$ is a lower bound for $\mathscr{C}$. 
We now show that there is a minimum invariant rectangle $\Sigma^{\prime}$ such that $\Sigma^{\prime} \prec \Sigma^{0}$. In view of Zorn's lemma, there is a maximum chain of $\mathscr{S}$ that contains $\Sigma^{0}$. Let $\mathscr{C}$ be one such maximum chain and let $\Sigma^{\prime}=\left[\hat{p}^{\prime}, \tilde{p}^{\prime}\right] \times\left[\widehat{N}^{\prime}, \tilde{N}^{\prime}\right]$ be a lower bound of $\mathscr{C}$. We show that $\Sigma^{\prime}$ is a minimum rectangle. Suppose this were not true. Without loss of generality, we may assume that $\hat{p}^{\prime}$ is strict with respect to $\Sigma^{\prime}$. Then there exists $\varepsilon>0$ such that

$$
\hat{p}^{\prime}>p_{s}+\varepsilon, \quad\left(\eta_{A}-\eta_{a}\right)\left(\hat{p}^{\prime}, N\right) \hat{p}^{\prime}\left(1-\hat{p}^{\prime}\right)>\varepsilon \text { for } N \in\left[\widehat{N}^{\prime}, \widetilde{N}^{\prime}\right] .
$$

By the continuity of $\eta_{A}-\eta_{a}$, there exists $\hat{p}^{\prime \prime}>\hat{p}^{\prime}$ such that

$$
p>p_{s}, \quad\left(\eta_{A}-\eta_{a}\right)(p, N) p(1-p)>0, \quad \text { for } p \in\left[\hat{p}^{\prime}, \hat{p}^{\prime \prime}\right], N \in\left[\widehat{N}^{\prime}, \tilde{N}^{\prime}\right] .
$$

Let $\Sigma(\tau)$ be defined by

$$
\Sigma(\tau)=\left[(1-\tau) \hat{p}^{\prime}+\tau \hat{p}^{\prime \prime}, \tilde{p}^{\prime}\right] \times\left[\widehat{N}^{\prime}, \tilde{N}^{\prime}\right] \text { for } \tau \in[0,1] .
$$

Then $\{\Sigma(\tau): \tau \in[0,1]\}$ is a family of invariant rectangles that satisfies all the conditions of [13, Theorem 3.1]. Since $\Sigma(0)=\Sigma^{\prime}, \Sigma(1) \subset \Sigma^{\prime}$, and $\Sigma(1) \neq \Sigma^{\prime}$, it follows that the union $\mathscr{C} \cup\{\Sigma(\tau) ; \tau \in[0,1]\}$ is a proper extension of $\mathscr{C}$. This contradicts the maximality of $\mathscr{C}$. The proof is complete. Q.E.D.

REMARK. An immediate consequence of Theorem 3.1 is that for each initial function $\left(p_{0}, N_{0}\right) \in[0,1] \times(0, \infty)$, the $\omega$-limit set of the solution is contained in the invariant rectangle $\Sigma_{0} \equiv[0,1] \times[\underline{N}, \bar{N}]$, where $\underline{N}$ and $\bar{N}$ are the minimum and maximum values of $N$ on the zero mean-fitness curve $\Gamma$ respectively. To see this, it suffices to show that every minimum invariant rectangle is a subset of $\Sigma_{0}$. Let $\Sigma^{\prime}=\left[\hat{p}^{\prime}, \tilde{p}^{\prime}\right] \times\left[\widehat{N}^{\prime}, \tilde{N}^{\prime}\right]$ be a minimum invariant rectangle. It is obvious that $\hat{p}^{\prime} \geq 0, \tilde{p}^{\prime} \leq 1$. Using the nonstrictness of $\widehat{N}^{\prime}$ and $\widetilde{N}^{\prime}$, we see that there exist $p_{1}, p_{2} \in\left[\hat{p}^{\prime}, \tilde{p}^{\prime}\right] \subset[0,1]$ such that

$$
\widehat{N}^{\prime} \eta\left(p_{1}, \widehat{N}^{\prime}\right)=0, \quad \tilde{N}^{\prime} \eta\left(p_{2}, \tilde{N}^{\prime}\right)=0 .
$$

Hence necessarily $\widehat{N}^{\prime} \geq \underline{N}, \widetilde{N}^{\prime} \leq \bar{N}$. This proves the assertion.

We now determine the attraction region of the uniform steady-state solution $\left(p_{s}, N_{s}\right)$ when it is $\left(0, K_{a a}\right),\left(1, K_{A A}\right)$, and $\left(p^{*}, N^{*}\right)$. For $\left(p_{s}, N_{s}\right)=\left(0, K_{A A}\right)$ we have the following result.

Theorem 3.2. Let assumption (A) hold and let $K_{A a}<K_{a a}$. Suppose $\Sigma=[0, \tilde{p}] \times$ $[\widehat{N}, \tilde{N}]$, where $\tilde{p}, \widehat{N}$, and $\widetilde{N}$ are positive constants satisfying the conditions

$$
\begin{gathered}
\tilde{p} \leq p^{*}, \quad \hat{N} \leq \phi(\tilde{p}), \quad \tilde{N} \geq K_{a a}, \quad \text { and } \\
\eta_{A}(p, N) \neq \eta_{a}(p, N) \quad \text { for }(p, N) \in \Sigma .
\end{gathered}
$$

Then every solution of Problem (1.2), (1.4) whose initial data satisfy the condition $\left(p_{0}(x), N_{0}(x)\right) \in[0, \hat{p}] \times[\widehat{N}, \tilde{N}]$ in $\Omega$ converges to $\left(0, K_{a a}\right)$ uniformly as $t \rightarrow \infty$.

Proof. We first show that $\Sigma$ is an invariant rectangle. This amounts to showing that the inequalities in (2.4) hold for $\hat{p}=0$ and $\tilde{p}, \widehat{N}, \widetilde{N}$ satisfying the above condition. Since the relation $K_{A a}<K_{a a}$ only occurs in type 3 systems, it follows from Lemma 2.1 that the function $\phi$ is monotone decreasing in $\left(0, p^{*}\right)$. Hence by 
the assumption $\widehat{N} \leq \phi(\tilde{p}), \tilde{N} \geq K_{a a}$ and the relation $\phi(0)=K_{a a}=N_{s}$ we have

$$
\tilde{N} \geq \phi(0)>\phi(p)>\phi(\tilde{p}) \geq \widehat{N}, \quad \text { for } p \in(0, \tilde{p}) .
$$

This implies that $\left(0, K_{a a}\right) \in \Sigma$. Since by assumption (A) and $K_{A a}<K_{a a}$,

$$
\eta_{A}\left(0, K_{a a}\right)=\eta_{A a}\left(K_{a a}\right)<0=\eta_{a}\left(0, K_{a a}\right),
$$

it follows from the assumption $\eta_{A} \neq \eta_{a}$ in $\Sigma$ that

$$
\eta_{A}(p, N)-\eta_{a}(p, N)<0 \text { for }(p, N) \in \Sigma,
$$

which implies the first inequality in (2.4) involving $\tilde{p}$. The second inequality in (2.4) is obvious because $\hat{p}=0$. The inequalities in (2.4) involving $\widehat{N}$ and $\widetilde{N}$ also follow from (3.2), since it implies that every point $(p, \widetilde{N})$ is above $\Gamma$ and every point $(p, \widehat{N})$ is below $\Gamma$ for $p \in(0, \tilde{p})$. Hence

$$
\eta(p, \widetilde{N})<0, \quad \eta(p, \widehat{N})>0 \quad \text { for } p \in(0, \tilde{p}) .
$$

This proves that $\Sigma$ is invariant.

We now show that every solution $(p, N)$ with the initial condition $\left(p_{0}, N_{0}\right) \in \Sigma$ converges to $\left(0, K_{a a}\right)$. Since by Theorem 3.1, every solution starting from $\Sigma$ has the $\omega$-limit set contained in a minimum invariant rectangle of $\Sigma$, it suffices to show that the only minimum invariant rectangle in $\Sigma$ is $\left\{\left(0, K_{a a}\right)\right\}$. Let $\Sigma^{\prime}=\left[\hat{p}^{\prime}, \tilde{p}^{\prime}\right] \times$ $\left[\widehat{N}^{\prime}, \tilde{N}^{\prime}\right] \subseteq \Sigma$ be a minimum rectangle. Then $\hat{p}^{\prime}, \tilde{p}^{\prime}, \widehat{N}^{\prime}, \widetilde{N}^{\prime}$ are nonstrict with respect to $\Sigma^{\prime}$. In particular, there is $N \in\left[\widehat{N}^{\prime}, \widetilde{N}^{\prime}\right]$ such that

$$
\left(\eta_{A}-\eta_{a}\right)\left(\tilde{p}^{\prime}, N\right) \tilde{p}^{\prime}\left(1-\tilde{p}^{\prime}\right)=0 .
$$

In view of relation (3.3) and $\tilde{p}^{\prime} \leq \tilde{p} \leq p^{*}$, it is necessary that $\tilde{p}^{\prime}=0$. Hence $\hat{p}^{\prime}=\tilde{p}^{\prime}=0$. Since $\widehat{N}^{\prime}$ and $\widetilde{N}^{\prime}$ are nonstrict in $\Sigma^{\prime}$, it follows that

$$
\eta\left(0, \widehat{N}^{\prime}\right)=0, \quad \eta\left(0, \widehat{N}^{\prime}\right)=0 .
$$

This implies that $\widehat{N}^{\prime}=\widetilde{N}^{\prime}=K_{a a}$. Hence $\Sigma^{\prime}=\left\{\left(0, K_{a a}\right)\right\}$ is the only possible minimum rectangle inside $\Sigma$. The proof is complete. Q.E.D.

The following result for the case $\left(p_{s}, N_{s}\right)=\left(1, K_{A A}\right)$ is similar to Theorem 3.2.

THEOREM 3.3. Suppose condition (A) and the relation $K_{A a}<K_{A A}$ hold. Let $\left(p_{s}, N_{s}\right)$ $=\left(1, K_{A A}\right)$ and let $\Sigma=[\hat{p}, 1] \times[\widehat{N}, \tilde{N}]$ where $\hat{p}, \hat{N}, \tilde{N}$ are positive constants satisfying the condition

$$
\begin{gathered}
\hat{p} \geq 0 \text { if } K_{A a} \geq K_{a a} \quad \text { or } \quad \hat{p} \geq p^{*} \text { if } K_{A a}<K_{a a}, \\
\hat{N} \leq \phi(\hat{p}), \quad \tilde{N} \geq K_{A A}, \\
\eta_{A}(p, N) \neq \eta_{a}(p, N) \quad \text { for }(p, N) \in \Sigma
\end{gathered}
$$

Then every solution $(p, N)$ with the initial condition $\left(p_{0}, N_{0}\right) \in \Sigma$ converges to $\left(1, K_{A A}\right)$ uniformly as $t \rightarrow \infty$. 
We omit the proof because it is similar to the proof of Theorem 3.2. The only major difference between Theorems 3.2 and 3.3 is that in Theorem 3.2, the inequality $K_{A a}<K_{a a}$ occurs only in type 3 systems, but in Theorem 3.3 the corresponding inequality $K_{A a}<K_{A A}$ occurs in both type 2 and type 3 systems. This difference is due to the assumption $K_{a a} \leq K_{A A}$. It does not affect the proof in any way.

We next give a condition for all the solutions of a type 2 system to converge to $\left(p_{s}, N_{s}\right)$ in the case $\left(p_{s}, N_{s}\right)=\left(1, K_{A A}\right)$.

Theorem 3.4. Let assumption (A) hold and let $K_{a a} \leq K_{A a} \leq K_{A A}$. Suppose

$$
\eta_{A}(p, N) \neq \eta_{a}(p, N)
$$

in the closed region $\mathscr{R}$ bounded by $\Gamma$ and the straight lines $p=0$ and $N=K_{A A}$. Then every solution $(p, N)$ of Problem (1.2), (1.4) converges to $\left(p_{s}, N_{s}\right)=\left(1, K_{A A}\right)$ uniformly in $\Omega$.

Proof. Since $\eta_{A}(p, N) \neq \eta_{a}(p, N)$ in $\mathscr{R}$, it follows that

$$
\eta_{A}(0, N) \neq \eta_{a}(0, N) \text { if } K_{a a} \leq N \leq K_{A A} .
$$

Observe that at the particular point $\left(0, K_{a a}\right)$

$$
\eta_{A}\left(0, K_{a a}\right)=\eta_{A a}\left(K_{a a}\right) \geq 0=\eta_{a}\left(0, K_{a a}\right) .
$$

Hence $\left(\eta_{A}-\eta_{a}\right)(0, N)>0$ if $K_{a a} \leq N \leq K_{A A}$. Thus by continuity there exists $\varepsilon>0$ such that

$$
\left(\eta_{A}-\eta_{a}\right)(p, N)>0 \text { for } p \in[0, \varepsilon] \text { and } N \in\left[K_{a a}-\varepsilon, K_{A A}+\varepsilon\right] .
$$

Let $\Sigma_{\delta}$ denote the rectangle $\Sigma_{\delta}=[\delta, 1] \times\left[K_{a a}-\varepsilon, K_{A A}+\varepsilon\right]$. Then by (3.4)

$$
\left(\eta_{A}-\eta_{a}\right)(\delta, N) \delta(1-\delta)>0 \text { for } N \in\left[K_{a a}-\varepsilon, K_{A A}+\varepsilon\right] .
$$

Using Lemma 2.1 and the assumption $K_{a a} \leq K_{A a} \leq K_{A A}$, we see that $\phi$ is monotone nondecreasing and

$$
K_{a a}=\phi(0) \leq \phi(p) \leq \phi(1)=K_{A A} \text { for } p \in[\delta, 1] .
$$

Hence by the monotone decreasing property of $\eta$ in $N$,

$$
\eta\left(p, K_{a a}-\varepsilon\right)>0, \quad \eta\left(p, K_{A A}+\varepsilon\right)<0 \quad \text { for } p \in[\delta, 1] .
$$

This proves that $\Sigma_{\delta}$ is invariant for each $\delta \in(0, \varepsilon]$.

To prove that every solution $(p, N)$ converges uniformly to the equilibrium $\left(1, K_{A A}\right)$, we show that $(p, N)$ enters into one of the invariant rectangles $\Sigma_{\delta}$ in finite time, and the only possible minimum invariant rectangle inside $\Sigma_{\delta}$ is the single point set $\left\{\left(1, K_{A A}\right)\right\}$. The convergence $(p, N) \rightarrow\left(1, K_{A A}\right)$ follows from these assertions and Theorem 3.1. To prove the first assertion we use the maximum principle and the remark following Theorem 3.1. Indeed, since by Lemma 2.1, $K_{a a}$ and $K_{A A}$ are the minimum and maximum values of $N$ on $\Gamma$ respectively, it follows that the $\omega$-limit set of the solution $(p, N)$ lies in the rectangle $[0,1] \times\left[K_{a a}, K_{A A}\right]$. Hence for some $T_{0}>0,(p, N)$ satisfies the relation

$$
\left(p\left(x, T_{0}\right), N\left(x, T_{0}\right)\right) \in[0,1] \times\left[K_{a a}-\varepsilon, K_{A A}+\varepsilon\right] \text { for } x \in \bar{\Omega} .
$$


Furthermore, by applying the maximum principle to the equation and boundary condition of $p$ in (1.2), (1.4), we see that $p(x, t)$ is positive for all $x \in \bar{\Omega}$ and $t>0$. Hence, for some positive $c_{0}$, it follows that $p\left(x, T_{0}\right) \geq c_{0}$ for all $x \in \bar{\Omega}$. By choosing $\delta \leq \min \left\{c_{0}, \varepsilon\right\}$, we have

$$
\left(p\left(x, T_{0}\right), N\left(x, T_{0}\right)\right) \in \Sigma_{\delta} \quad \text { for } x \in \bar{\Omega} .
$$

Hence $(p, N)$ enters into $\Sigma_{\delta}$ before $t=T_{0}$.

It remains to show that the only possible minimum invariant rectangle inside $\Sigma_{\delta}$ is the single point set $\left\{\left(1, K_{A A}\right)\right\}$. Let $\Sigma^{\prime}=\left[\hat{p}^{\prime}, \tilde{p}^{\prime}\right] \times\left[\widehat{N}^{\prime}, \tilde{N}^{\prime}\right]$ be a minimum invariant rectangle inside $\Sigma_{\delta}$. Then by nonstrictness of $\hat{p}^{\prime}, \tilde{N}^{\prime}$ it follows that

$$
\eta\left(p, \widetilde{N}^{\prime}\right)=0, \quad\left(\eta_{A}-\eta_{a}\right)\left(\hat{p}^{\prime}, N\right) \hat{p}^{\prime}\left(1-\hat{p}^{\prime}\right)=0
$$

for some $p \in\left[\hat{p}^{\prime}, \tilde{p}^{\prime}\right]$ and $N \in\left[\widehat{N}^{\prime}, \widetilde{N}^{\prime}\right]$. The first equation implies that $\widehat{N}^{\prime} \leq K_{A A}$ and the second equation implies that either $\hat{p}^{\prime}=1$ or $\left(\hat{p}^{\prime}, N\right) \notin \mathscr{R}$. The latter is impossible because it would lead to the relation $N<\phi\left(\hat{p}^{\prime}\right)$, which implies

$$
\widehat{N}^{\prime} \leq N<\phi\left(\hat{p}^{\prime}\right) \leq \phi(p) \text { for } p \in\left[\hat{p}^{\prime}, \tilde{p}^{\prime}\right]
$$

by the monotone nondecreasing property of $\phi$. Hence we have $\eta\left(p, \widehat{N}^{\prime}\right)>0$ for all $p \in\left[\hat{p}^{\prime}, \tilde{p}^{\prime}\right]$, which contradicts the nonstrictness of $\widehat{N}^{\prime}$ with respect to $\Sigma^{\prime}$. Thus it is necessary that $\hat{p}^{\prime}=\tilde{p}^{\prime}=1$. Since $\widehat{N}^{\prime}$ is nonstrict with respect to $\Sigma^{\prime}$, it follows that $\eta\left(1, \widehat{N}^{\prime}\right)=0$. Hence $\widehat{N}^{\prime}=\phi(1)=K_{A A}$ and consequently $\widetilde{N}^{\prime}=\widehat{N}^{\prime}=K_{A A}$. This proves the assertion $\Sigma^{\prime}=\left\{\left(1, K_{A A}\right)\right\}$. The proof is complete. Q.E.D.

We next discuss the attraction region of $\left(p_{s}, N_{s}\right)$ when $\left(p_{s}, N_{s}\right)=\left(p^{*}, N^{*}\right)$.

Theorem 3.5. Let condition (A) hold and let $K_{A a} \geq K_{A A}$. Suppose $\left(p_{s}, N_{s}\right)=$ $\left(p^{*}, N^{*}\right)$. Then every solution $(p, N)$ of Problem (1.2), (1.3) converges uniformly to $\left(p_{s}, N_{s}\right)$ whenever the initial function $\left(p_{0}, N_{0}\right) \in E$.

Proof. Observe that $K_{A a} \geq K_{A A}$ holds for type 1 and 2 systems. For the latter type systems, $\left(p^{*}, N^{*}\right)$ exists only if $K_{a a}=K_{A a}=K_{A A}$. The proof of the theorem for this case is straightforward. Recall that since none of (2.7), (2.9), (2.13) holds, $\left(p_{s}, N_{s}\right)$ is asymptotically stable. Hence it suffices to show that for each solution $(p, N)$ of Problem (1.2), (1.4) there is a positive constant $T_{0}$ such that $\left(p\left(\cdot, T_{0}\right), N\left(\cdot, T_{0}\right)\right)$ is in a small neighborhood of $\left(p_{s}, N_{s}\right)$. Since $K_{a a}=K_{A a}=K_{A A}$ implies that the zero mean-fitness curve $\Gamma$ is the horizontal line segment $N=N^{*} \equiv K_{A A}$ over the interval $p \in[0,1]$, by the remark following Theorem 3.1, the $\omega$-limit set $\omega(p, N)$ lies in the set

$$
0 \leq p(x) \leq 1, \quad N(x) \equiv N^{*}, \quad x \in \Omega .
$$

Let $\left(p_{1}, N_{1}\right) \in \omega(p, N)$ and let $\left(p^{\prime}(\cdot, t), N^{\prime}(\cdot, t)\right)$ be the solution of (1.2), (1.4) such that $\left(p^{\prime}(\cdot, 0), N^{\prime}(\cdot, 0)\right)=\left(p_{1}, N_{1}\right)$. Then by the invariance property of $\omega$ limit sets, the entire orbit $\left\{\left(p^{\prime}(\cdot, t), N^{\prime}(\cdot, t)\right): t \in \mathbb{R}\right\}$ is contained in $\omega(p, N)$. Furthermore, since $N^{\prime}(x, t)=N^{*}$ for all $t \geq 0, x \in \Omega$ and $p^{\prime}(x, t)$ is a solution 
of the problem

$$
p_{t}-\Delta p=0 \quad \text { in } \Omega \times(0, \infty),\left.\quad p\right|_{\partial \Omega}=p^{*},
$$

it is easy to see that $\left(p^{\prime}, N^{\prime}\right)$ converges to $\left(p^{*}, N^{*}\right)$ uniformly as $t \rightarrow \infty$. Hence, for each $\varepsilon>0$ there is $T_{0}^{\prime}>0$ such that $\left(p^{\prime}\left(\cdot, T_{0}^{\prime}\right), N^{\prime}\left(\cdot, T_{0}^{\prime}\right)\right)$ lies in the $\varepsilon / 2$ neighborhood of $\left(p^{*}, N^{*}\right)$. By the definition of $\omega$-limit set, there is a $T_{0}>0$ such that $\left(p\left(\cdot, T_{0}\right), N\left(\cdot, T_{0}\right)\right)$ is in the $\varepsilon$-neighborhood of $\left(p^{*}, N^{*}\right)$. This proves the convergence of $(p, N)$ to the uniform steady-state solution $\left(p_{s}, N_{s}\right)$.

We now prove the theorem for type 1 systems. By Lemma 2.1, $\phi_{A}$ is decreasing, $\phi_{a}$ is increasing, and $N^{*}$ is the maximum of the function $\phi$ in $p \in[0,1]$. We first show that if $\phi(p) \leq N \leq N^{*}$ then

$$
\begin{array}{ll}
\left(\eta_{A}-\eta_{a}\right)(p, N)>0 & \text { for } p \in\left[0, p^{*}\right), \\
\left(\eta_{A}-\eta_{a}\right)(p, N)<0 & \text { for } p \in\left(p^{*}, 1\right] .
\end{array}
$$

Since, by (2.11),

$$
N^{*}=\phi_{A}\left(p^{*}\right)=\phi_{a}\left(p^{*}\right)=\phi\left(p^{*}\right),
$$

it follows from the monotonicity of $\phi_{A}$ and $\phi_{a}$ that $N^{*}<\phi_{A}(p)$ if $0 \leq p<p^{*}$ and $N^{*}<\phi_{a}(p)$ if $p^{*}<p \leq 1$. Hence in the case where $\phi(p) \leq N \leq N^{*}, p \in\left[0, p^{*}\right)$, we have $\eta(p, N) \leq 0$ and $\eta_{A}(p, N)>0$. Using the relation

$$
\eta(p, N)=p \eta_{A}(p, N)+(1-p) \eta_{a}(p, N),
$$

we see that $\eta_{a}(p, N) \leq 0$, which implies the first inequality in (3.5). The second inequality is proven analogously.

We next construct an invariant rectangle $\Sigma_{1}$ as follows. Since, by (3.5), $\left(\eta_{A}-\eta_{a}\right)(0, N)>0$ for $K_{a a} \leq N \leq N^{*}$, it follows from the continuity of $\eta_{A}-\eta_{a}$ that for some $\varepsilon_{1}>0$

$$
\left(\eta_{A}-\eta_{a}\right)(p, N)>0 \quad \text { if } 0 \leq p \leq \varepsilon_{1} \text { and } K_{a a}-\varepsilon_{1} \leq N \leq N^{*}+\varepsilon_{1} .
$$

Let $\Sigma_{1}=\left[\delta_{1}, 1\right] \times\left[K_{a a}-\varepsilon_{1}, N^{*}+\varepsilon_{1}\right]$, where $\delta_{1} \in\left(0, \varepsilon_{1}\right)$ is to be determined later. To see that $\Sigma_{1}$ is invariant, we verify the inequalities in (2.4) for $\hat{p}=\delta_{1}$, $\widehat{N}=K_{a a}-\varepsilon_{1}$, and $\widetilde{N}=N^{*}+\varepsilon_{1}$. The inequality for $\hat{p}=\delta_{1}$ follows directly from relation (3.6). As for the inequalities involving $\widehat{N}$ and $\widetilde{N}$, we use Lemma 2.1 and the assumption $K_{a a} \leq K_{A A}$ to obtain

$$
K_{a a} \leq \phi(p) \leq N^{*} \text { for } p \in[0,1] .
$$

This implies that

$$
\eta\left(p, K_{a a}-\varepsilon_{1}\right)>0 \text { and } \eta\left(p, N^{*}+\varepsilon_{1}\right)<0 .
$$

Hence $\Sigma_{1}$ is invariant.

We show that every solution $(p, N)$ enters into $\Sigma_{1}$ in finite time for some suitable $\delta_{1}$. Indeed, since by (3.7) $K_{a a}$ and $N^{*}$ are the minimum and maximum values of $N$ on $\Gamma$ respectively, the remark following Theorem 3.1 shows that every solution converges uniformly in the rectangle $[0,1] \times\left[K_{a a}, N^{*}\right]$. Hence there exists $T_{1}>0$ 
such that

$$
K_{a a}-\varepsilon_{1} \leq N\left(x, T_{1}\right) \leq N^{*}+\varepsilon_{1} \text { for } x \in \bar{\Omega} .
$$

In view of the strong maximum principle, $p(x, t)>0$ for all $x \in \bar{\Omega}, t>0$. Hence for some small $\delta_{1}>0, p\left(x, T_{1}\right) \geq \delta_{1}$ in $\bar{\Omega}$. Using this $\delta_{1}$, we have

$$
\left(p\left(x, T_{1}\right), N\left(x, T_{1}\right)\right) \in \Sigma_{1} \text { for } x \in \bar{\Omega} .
$$

The assertion is proven.

We construct another invariant rectangle $\Sigma_{2}$ such that $0<p<1$ in $\Sigma_{2}$ and $(p, N)$ also enters $\Sigma_{2}$ in finite time. Since $\Sigma_{1}$ is invariant, by Theorem 3.1 , there exists a minimum invariant rectangle $\Sigma_{1}^{\prime}=\left[\hat{p}^{\prime}, \tilde{p}^{\prime}\right] \times\left[\widehat{N}^{\prime}, \widetilde{N}^{\prime}\right]$ containing the $\omega$-limit set of $(p, N)$. We show that $\widehat{N}^{\prime} \geq K_{A A}$. Suppose by contradiction that $\widehat{N}^{\prime}<K_{A A}$. Since $\widehat{N}^{\prime}$ is nonstrict with respect to $\Sigma_{1}^{\prime}$, there is $p_{1} \in\left[\hat{p}^{\prime}, \tilde{p}^{\prime}\right]$ such that $\eta\left(p_{1}, \widehat{N}^{\prime}\right)=$ 0 . Hence $K_{A A}>\widehat{N}^{\prime}=\phi\left(p_{1}\right)$. Since $\phi(p)$ is increasing in $\left[0, p^{*}\right)$ and decreasing in $\left(p^{*}, 1\right]$, it follows that $\phi(p) \geq \phi(1)=K_{A A}$ in $\left[p^{*}, 1\right]$. Thus $\hat{p}^{\prime} \leq p_{1}<p^{*}$, which leads to $\phi\left(\hat{p}^{\prime}\right) \leq \phi\left(p_{1}\right)=\widehat{N}^{\prime}$. Hence by $(3.5),\left(\eta_{A}-\eta_{a}\right)\left(\hat{p}^{\prime}, N\right)>0$ for all $N \in\left[\hat{N}^{\prime}, \tilde{N}^{\prime}\right]$. This contradicts the nonstrictness of $\hat{p}^{\prime}$ with respect to $\Sigma_{1}^{\prime}$. Therefore $\widehat{N}^{\prime} \geq K_{A A}$. Take $p_{2} \in\left[0, p^{*}\right)$ such that $\phi\left(p_{2}\right)=K_{A A}$, whose existence follows from the intermediate value theorem and the relations $\phi(0)=K_{a a} \leq K_{A A}$ and $\phi\left(p^{*}\right)=$ $N^{*}>K_{A A}$. It is necessary that $\hat{p}^{\prime}>p_{2}$, for if $\hat{p}^{\prime} \leq p_{2}$, then $\phi\left(\hat{p}^{\prime}\right) \leq \phi\left(p_{2}\right)=K_{A A}$, and hence for each $N \geq \widehat{N}^{\prime} \geq K_{A A}$ the relation (3.5) would give $\left(\eta_{A}-\eta_{a}\right)\left(\hat{p}^{\prime}, N\right)>0$, which shows that $\hat{p}^{\prime}$ cannot be nonstrict with respect to $\Sigma_{1}^{\prime}$. Applying (3.5) with $p=p_{2}$ and $p=1$, we have

$$
\left(\eta_{A}-\eta_{a}\right)\left(p_{2}, N\right)>0 \text { and }\left(\eta_{A}-\eta_{a}\right)(1, N)<0 \text { for } N \in\left[K_{A A}, N^{*}\right]
$$

By continuity, there is $\varepsilon_{2}>0$ such that

$$
\begin{array}{ll}
\left(\eta_{A}-\eta_{a}\right)(p, N)>0 & \text { if } p_{2} \leq p \leq p_{2}+\varepsilon_{1}, \\
\left(\eta_{A}-\eta_{a}\right)(p, N)<0 & \text { if } 1-\varepsilon_{1} \leq p \leq 1
\end{array}
$$

whenever $K_{A A}-\varepsilon_{2} \leq N \leq N^{*}+\varepsilon_{2}$. Define $\Sigma_{2}$ by

$$
\Sigma_{2}=\left[p_{2}+\delta_{2}, 1-\delta_{2}\right] \times\left[K_{A A}-\varepsilon_{2}, N^{*}+\varepsilon_{2}\right],
$$

where $\delta_{2} \in\left(0, \varepsilon_{2}\right)$ is a suitable constant to be determined later. We show that for each $\delta_{2} \in\left(0, \varepsilon_{2}\right), \Sigma_{2}$ is an invariant rectangle, and for each solution $(p, N)$, there is $\delta_{2}$ such that $(p, N)$ enters $\Sigma_{2}$ in finite time. We first check that the inequalities in (2.4) hold for $\hat{p}=p_{2}+\delta_{2}, \widehat{N}=K_{A A}-\varepsilon_{2}, \tilde{p}=1-\delta_{2}$, and $\tilde{N}=N^{*}+\varepsilon_{2}$. The inequalities involving $\hat{p}$ and $\tilde{p}$ follow immediately from (3.8). Since $\phi\left(p_{2}\right)=K_{A A}$, using the monotonicity of $\phi$ it follows that

$$
\begin{array}{ll}
\phi(p)>\phi\left(p_{2}\right)=K_{A A} & \text { if } p_{2}+\delta \leq p \leq p^{*} \\
\phi(p) \geq \phi\left(1-\delta_{2}\right)>\phi(1)=K_{A A} & \text { if } p^{*} \leq p \leq 1-\delta_{2} .
\end{array}
$$

Hence $\eta\left(p, K_{A A}-\varepsilon_{2}\right)>0$ for all $p \in\left[p_{2}+\delta_{2}, 1-\delta_{2}\right]$. On the other hand, since $N^{*}$ is the maximum value of $\phi$, it follows that $\phi(p)<N^{*}+\varepsilon_{2}$, which is equivalent to 
$\eta\left(p, N^{*}+\varepsilon_{2}\right)<0$. Therefore the inequalities involving $\widehat{N}$ and $\widetilde{N}$ are also satisfied. Hence $\Sigma_{2}$ is invariant. We next show that if $(p, N)$ is a solution then there are constants $\delta_{2} \in\left(0, \varepsilon_{2}\right)$ and $T_{2}>0$ such that

$$
\left(p\left(x, T_{2}\right), N\left(x, T_{2}\right)\right) \in \Sigma_{2} \text { for } x \in \bar{\Omega} .
$$

Since $(p, N)$ converges uniformly in $\Sigma_{1}^{\prime}$ as $t \rightarrow \infty$, it follows from the relations $\hat{p}^{\prime}>p_{2}, \hat{N}^{\prime} \geq K_{A A}$, and $\tilde{N}^{\prime} \leq N^{*}$ that for some $T_{2}>0$

$$
p_{2}<p\left(x, T_{2}\right), \quad K_{A A}-\varepsilon_{2} \leq N\left(x, T_{2}\right) \leq N^{*}+\varepsilon_{2} \quad \text { for } x \in \bar{\Omega} .
$$

Furthermore, using the strong maximum principle, we see that $p(x, t)<1$ for all $x \in \bar{\Omega}, t>0$. Hence there exists $\delta_{2}>0$ such that $\delta_{2} \leq \varepsilon_{2}$ and

$$
p_{2}+\delta_{2} \leq p\left(x, T_{2}\right) \leq 1-\delta_{2} .
$$

With this $\delta_{2}$ we see that (3.9) holds.

Finally, we show that the only possible minimum rectangle in $\Sigma_{2}$ is the singlepoint set $\left\{\left(p^{*}, N^{*}\right)\right\}$. Once this assertion is proven, the convergence of $(p, N)$ to $\left(p^{*}, N^{*}\right)$ is a consequence of Theorem 3.1. Let $\Sigma^{\prime \prime}=\left[\hat{p}^{\prime \prime}, \tilde{p}^{\prime \prime}\right] \times\left[\widehat{N}^{\prime \prime}, \tilde{N}^{\prime \prime}\right]$ be a minimum invariant rectangle inside $\Sigma_{2}$. Then $\hat{p}^{\prime \prime}>0, \tilde{p}^{\prime \prime}<1$, and $\hat{p}^{\prime \prime}, \tilde{p}^{\prime \prime}, \widehat{N}^{\prime \prime}, \widetilde{N}^{\prime \prime}$ are nonstrict in $\Sigma^{\prime \prime}$. We first show that $\widehat{N}^{\prime \prime}=N^{*}$. Since $\widehat{N}^{\prime \prime}$ is nonstrict in $\Sigma^{\prime \prime}$, there exists $p_{3} \in\left[\hat{p}^{\prime \prime}, \tilde{p}^{\prime \prime}\right]$ such that $\eta\left(p_{3}, \widehat{N}^{\prime \prime}\right)=0$. Hence $\widehat{N}^{\prime \prime}=\phi\left(p_{3}\right)$. If $p_{3} \leq p^{*}$, then by the monotonicity of $\phi, \phi\left(\hat{p}^{\prime \prime}\right) \leq \phi\left(p_{3}\right)=\widehat{N}^{\prime \prime}$. In view of $(3.5)$, if $\hat{p}^{\prime \prime} \neq p^{*}$ then $\left(\eta_{A}-\eta_{a}\right)\left(\hat{p}^{\prime \prime}, N\right)>0$ for each $N \in\left[\widehat{N}^{\prime \prime}, \tilde{N}^{\prime \prime}\right] \subseteq\left[\widehat{N}^{\prime \prime}, N^{*}\right]$, contradicting the assumption that $\hat{p}^{\prime \prime}$ is nonstrict in $\Sigma^{\prime \prime}$. Hence $\hat{p}^{\prime \prime}=p_{3}=p^{*}$. Similarly $\tilde{p}^{\prime \prime}=p_{3}=p^{*}$ if $p_{3} \geq p^{*}$. Hence in each case we have

$$
\widehat{N}^{\prime \prime}=\phi\left(p_{3}\right)=\phi\left(p^{*}\right)=N^{*} \text {. }
$$

This proves that $\widehat{N}^{\prime \prime}=\widetilde{N}^{\prime \prime}=N^{*}$. Finally, using the fact that $\hat{p}^{\prime \prime}$ and $\tilde{p}^{\prime \prime}$ are nonstrict in $\Sigma^{\prime \prime}$, we see that

$$
\left(\eta_{A}-\eta_{a}\right)\left(\hat{p}^{\prime \prime}, N^{*}\right)=0, \quad\left(\eta_{A}-\eta_{a}\right)\left(\tilde{p}^{\prime \prime}, N^{*}\right)=0 .
$$

Hence, by (3.5) it is necessary that $\hat{p}^{\prime \prime}=\tilde{p}^{\prime \prime}=p^{*}$. This leads to $\Sigma^{\prime \prime}=\left\{\left(p^{*}, N^{*}\right)\right\}$. Q.E.D.

Acknowledgment. The author would like to thank Professor Roger Lui for helpful conversations.

\section{REFERENCES}

[1] H. Amann, Fixed point equations and nonlinear eigenvalue problems in ordered Banach spaces, SIAM Review 18, 620-709 (1976)

[2] J. W. Bebernes, K.-N. Chueh, and W. Fulks, Some applications of invariance for parabolic systems, Indiana Univ. Math. J. 28, 269-277 (1979)

[3] P. N. Brown, Decay to uniform states in ecological interactions, SIAM J. Appl. Math. 38, 22-37 (1980)

[4] E. N. Dancer, On the indices of fixed points of mappings in cones and applications, J. Math. Anal. Appl. 91, 131-151 (1982) 
[5] L. R. Ginzburg, The equilibrium and stability for $n$ alleles under the density-dependent selection, J. Theor. Biol. 68, 545-550 (1977)

[6] L. Li, Coexistence theorems of steady states for predator-prey interacting systems, Trans. Amer. Math. Soc. 305, 143-166 (1988)

[7] R. Lui, Convergence to constant equilibrium for a density-dependent selection model with diffusion, J. Math. Biol. 26, 583-592 (1988)

[8] R. Lui, Positive solutions of an elliptic system arising from a model in evolutionary ecology, J. Math. Biol. 29, 239-250 (1991)

[9] R. Lui and J. F. Selgrade, Density-dependent selection migration model with nonmonotone fitness functions, J. Math. Biol. 31, 717-734 (1993)

[10] C. V. Pao, Nonlinear Parabolic and Elliptic Equations, Plenum, New York, 1992

[11] M. G. Protter and H. F. Weinberger, Maximum Principle in Differential Equations, Prentice-Hall, Englewood Cliffs, New Jersey, 1967

[12] W. H. Ruan, Convergence to constant states in a population genetic model with diffusion, Nonlinear Analysis, TMA (to appear)

[13] W. H. Ruan, One-parameter family of invariant sets for non-weakly coupled nonlinear parabolic systems, J. Math. Anal. Appl. 189, 763-780 (1995)

[14] W. H. Ruan, Multiple steady-state solutions of nonlinear parabolic systems in convex invariant sets, Nonlinear Analysis, TMA (to appear)

[15] W. H. Ruan and W. Feng, On the fixed point index and multiple steady-state solutions of reaction-diffusion systems, Differential and Integral Equations 8, 371-391 (1995)

[16] J. F. Selgrade and G. Namkoong, Dynamical behavior of differential equation models of frequency and density dependent populations, J. Math. Biol. 19, 133-146 (1984)

[17] J. Smoller, Shock Waves and Reaction-Diffusion Equations, Springer-Verlag, New York, Heidelberg, Berlin, 1983

[18] M. Wang, Z. Li, and Q. Ye, The existence of positive solutions for semilinear elliptic systems, Qualitative Aspects and Applications of Nonlinear Evolution Equations (Proc. School, Trieste, 1990), World Scientific, 1991, pp. 256-269 (P. DeMottoni and T. T. Li, eds.) (See also Acta Sci. Natur. Univ. Pekinensis 28, 36-49.)

[19] L.-Y. Tsai, Existence of solution of nonlinear elliptic systems, Bull. Inst. Math. Acad. Sinica 5, 219-247 (1977) 